\title{
Reducing the incidence of burn injuries to Indigenous Australian children
}

\author{
Roy M Kimble and Bronwyn R Griffin
}

Burns are a specific health burden, but understanding the detail is vital to finding solutions

It is undisputed that Aboriginal and Torres Strait Islander (Indigenous Australian) children are over-represented in statistics for injury and death caused by trauma. The incidence of each of the major mechanisms of fatal trauma in Australian children — drowning and low speed vehicle run-overs — is higher among Indigenous children.1,2 Burn injuries are also more prevalent among Indigenous children. $\underline{3}$

In this issue of the MJA, Möller and his colleagues report a population data linkage study they undertook in New South Wales. 4 Their results not only confirm that the incidence of hospitalisation of children for burn injuries is higher among Indigenous than non-Indigenous children. The authors also found that the proportion of burn injuries affecting more than $20 \%$ of total body surface area (TBSA) was greater for Indigenous than for non-Indigenous children, as was that of burns to the feet or ankles; that the incidence of being treated in a tertiary burns facility was lower and their median overall hospital stay longer for Indigenous children; and that they were less likely to undergo surgery, but more frequently received treatment from allied health professionals. This important epidemiological study not only supports the hypothesis that burn injuries constitute a significant health burden in Indigenous children, it is also the prelude to a much larger prospective study. $\underline{5}$ Paediatric burns services throughout Australia are currently collaborating in a study funded by the National Health and Medical Research Council to examine the journey of the Indigenous child with a burn injury through the health system, including pre-hospital care and outpatient follow-up. $\underline{5}$

The report by Möller and co-authors is initially somewhat disturbing, but more detailed analysis identifies factors that explain some of the disparities described. The proportion of Indigenous Australians living in rural and remote geographic locations, and therefore a long distance from tertiary burns facilities, is higher than for other Australians. It is consequently not surprising that many Indigenous children are treated in their local hospital, which has the advantage of keeping the family unit closer to home, with clear psychosocial and financial benefits. With the advent of telehealth services linking major burns services and local hospitals, and the application of digital photography to record wound status at each dressing change, a high standard of care can now be achieved even in remote locations. $\underline{6}, \underline{7}$

Whether a child needs to be admitted to hospital for a burn injury depends on many factors apart from the proportion of TBSA burned. One-third of children are admitted because of the impact of the injury on their family, not because immediate treatment of the burn is needed. $\underline{8}$ It is often in the interest of the Indigenous child and family to be admitted to hospital when factors such as remoteness of the family home and socio-economic disadvantage would prevent the families traveling to outpatient appointments for dressing changes. Not only is admission to hospital more likely under these circumstances, the duration of stay will also be longer. 
The region of the body affected by a burn is very much related to the mechanism of injury. For example, hot beverage scalds usually affect the face, neck and torso, whereas burns by hot embers and ash from campfires and burn-offs typically affect feet and ankles. Indigenous children have different patterns of burn injury types to other Australian children because of cultural and socio-economic differences. The higher proportion of foot and ankle burns in the report by Möller and colleagues is possibly explained by a higher incidence of campfire burns to Indigenous children.

The estimated TBSA burned is probably the greatest source of inaccuracy when documenting a burn injury. $\underline{9}$ Areas of superficial burn (erythema only) are often erroneously included, leading to grossly overestimating the extent of the burn. Burn depth can also progress with time, but the TBSA is often not re-calculated, so that the initial estimate is the only value documented by hospital coders. Overestimation of burn extent would probably occur more frequently in non-tertiary facilities. Lund and Browder charts have traditionally been employed for calculating TBSA, but they are cumbersome to use and should therefore be replaced by mobile phone apps that accurately estimate TBSA by digitally shading on the screen the areas affected. The New South Wales Institute of Trauma has developed an app for this purpose that is free, quick and easy to use; the age and weight of the child are entered, and the degree of fluid resuscitation required (using the Parkland formula) is also calculated. $\underline{10}$

People from populations with darker skin colour are reported to re-epithelialise burn injuries up to $25 \%$ more quickly than those from populations with lighter skin.11 However, darker skin has a higher propensity for hypertrophic scarring, explaining why the Indigenous children in this study had fewer operations for skin grafting, but significantly greater requirements for management by allied health professionals.

Preventing burns must be part of any intervention to reduce the burden of burn injuries in Indigenous children, alongside optimal first aid. Campaigns to prevent burn injuries will only be successful if they are targeted at specific populations that are at greater risk, and it is important they include collaboration between injury prevention advocates, Indigenous leaders, and health care workers. 